\title{
गेंịj talentabipis
}

International Journal of

NASOPHARYNGEAL CARCINOMA

Journal homepage: ijnpc.usu.ac.id

\section{CLINICAL SIGNIFICANCE OF PLASMA MIR-21, MIR-141, MIR-29C, AND MIR-BART7 IN PATIENTS WITH LOCALLY ADVANCED NASOPHARYNGEAL CANCER AND THEIR ALTERATIONS AFTER CHEMORADIATION THERAPY}

\author{
Cita Herawati ${ }^{*}$ \\ ${ }^{I}$ Department of Ear, Nose, and Throat, Dharmais National Cancer Hospital, Jakarta
}

\begin{abstract}
Introduction: Plasma microRNAs (miRNAs) are biological markers that have been extensively studied in cancer, including nasopharyngeal cancer (NPC). The clinical significance of miRNA in NPC patients in Indonesia has never been studied.

Objective: This study was aimed to know the expression of plasma miRNAs in NPC patients (miR-21, miR-29c, miR 141dan miR-BART7) and their relationship with clinicopathological characteristics and treatment response.

Method: This was a cohort, longitudinal study among locally advanced NPC patients (stage IIB-IVB) in Dharmais Cancer Hospital, Jakarta. miRNA expression was assayed using quantitative real-time polymerase chain reaction (qRT-PCR) technique. Four miRNAs were evaluated, i.e., miR-21, miR-29c, miR-141, and EBVmiR-BART7. The results were normalized against a reference gene, miR-16.

Result: A total of 52 patients and 10 normal subjects were enrolled in this study; 17 of them completed treatment. Patients' mean age was 45.1+12.53 (14-68) years. The ratio between men and women was 3:1. MiR-21 and miR-29c could be detected in all subjects; miR-141 was detected in $22(42.3 \%)$ and EBV-miR-BART7 in 26 $(50 \%)$ subjects. There was no significant difference between miR-21 or miR-29c expression between before and after therapy. However, miR-21 expressions tend to decrease in a patient with complete response (CR) $(4.13+3.65$ vs. $2.74+3.23 ; p=0.650)$ and tend to increase in patients with partial response (PR) $(3.00+5.86$ vs $8.77+8.43 ; \mathrm{p}=0.465)$. There was no difference of miR-29c expression between CR and PR patients.

Conclusion: Our study shows that not all miRNA can be detected in the plasma of NPC patients. Levels of miRNA expressions in these locally advanced patients are similar. Expression of miR-21 is potentially used as a biomarker of evaluating treatment response in NPC patients.
\end{abstract}

\author{
Article Info \\ Keywords \\ miR-21, miR-29c, miR-141, miR-BART7, \\ nasopharyngeal cancer
}

\section{*Corresponding author}

Address: Slipi, Jl. Letjen. S. Parman No.84-86, RT.4/RW.9, Kota Bambu Sel, Kec. Palmerah, Kota Jakarta Barat, DKI Jakarta 11420, Indonesia

e-mail: citaherawati@gmail.com

\section{INTRODUCTION}

Nasopharyngeal carcinoma (NPC) is cancer with a unique distribution. In 2008 , more than 84,000 new cases occurred annually, representing about $0.7 \%$ of all cancers worldwide. Southern China and Southeast Asian countries, including Indonesia, are regions with the highest incidence of NPC [1]. In Indonesia, NPC is the most frequent head and neck cancer with male predominance and affects patients in their young [2].

NPC is radiosensitive cancer, but $60 \%$ to $70 \%$ of patients came in advanced stage due to unspecific symptoms [3]. Because of the final anatomical structure of nasopharynx, monitoring of treatment results and recurrences are often not optimally achieved; therefore, non-invasive and specific biomarkers are essential to identify high-risk individuals and predict treatment response.

One of the most studied biomarkers in the world is the expression level of plasma microRNA started in 2006. MicroRNA (miRNA) is a small, non-coding RNA molecule-sized 20-22 nucleotides, which regulates the expression of many genes post-transcriptionally. MiRNA is an important regulator of various cellular processes, including cell proliferation, differentiation, apoptosis, survival, motility, and morphogenesis.

In a search for an effective biomarker in NPC, it tested three human miRNA and one EBV miRNA that has been studied elsewhere with convincing results.

MiR-21 is one of the most extensively studied miRNA. Its expression is initiated by hypoxia and is upregulated in many cancers [4]. MiR-21 acts as an oncomir because of its capability to inhibit tumor suppressor genes, induce tumor growth, invasion, and metastasis [5, 6]

The mir-29c expression is down-regulated in NPC tissues and metastasis. It has a tumor suppressor effect through its effect on the extracellular matrix [7]. MiR141 was reported to be up-regulated in NPC tissues when compared to normal nasopharyngeal epithelia and may affect cell cycle, apoptosis, cell growth, migration and invasion of NPC cells [8]. MiR-BART7 was one of Epstein-Barr virus (EBV)-derived miRNAs, which were reported to be overexpressed in the plasma of undifferentiated NPC patients compared to healthy individuals [9].

This study was aimed to evaluate the expression of miR-21, miR-29c, miR141 and miR-BART7 in the plasma of NPC patients and to explore their relationship with clinical characteristics and treatment response.

\section{MATERIAL AND METHODS}

\subsection{Study design and subjects}

The study design was a longitudinal cohort study in NPC patients in Dharmais Cancer Hospital (DCH) between December 2013 and May 2015. Subjects were recruited from ENT Department, Dharmais Cancer Hospital ((DCH), whereas plasma miRNA expressions were assessed in the Biomolecular Laboratory of the Faculty of Medicine, Gadjah Mada University, Yogyakarta. Diagnosis of NPC was confirmed histopathologically from biopsy specimens of nasopharyngeal exploration.

\subsection{Preliminary study to find a reference gene}

Before evaluating miRNA expressions in true clinical patients, it has been performed a preliminary study to set laboratory protocols and to select a reference gene. Plasma specimens were obtained from several patients and were tested for miR-16, miR-21, miR-145, miR-155, and U6 small nuclear (sn) RNAs. Methods of RNA extraction and miRNA expression quantification were given in detail below. Amplification curve, melt curve, and melt peak of each miRNA were obtained and analyzed. 


\subsection{RNA extraction and quantitative real-time polymerase chain reaction}

Five $\mathrm{mL}$ of blood specimens were drawn from NPC patients and healthy subjects and stored in EDTA-containing vacutainers. Blood specimens were centrifuged to obtain the plasma and then were stored at $-80 \mathrm{oC}$ before further processes.

Total plasma RNA was extracted using a commercial kit (miRCURY RNA Isolation Kit-Biofluid, Exiqon, USA) following the manufacturer's protocol. After isolation, RNA was reversely transcribed to cDNA using Universal cDNA Synthesis Kit II, 8-64 rxns, Exiqon, USA). Samples were then amplified and quantified using the real-time polymerase chain reaction (qRT-PCR) technique (ExiLent SYBR Green Master Mix, $2.5 \mathrm{~mL}$, Cat. No. \#203402, Exiqon, USA). Identification of miRNA sequence was done using commercial kits (miRCURY LNA LNA ${ }^{\mathrm{TM}}$ Universal RT microRNA PCR, Exiqon, USA) as follows:

Table 1. Identification of miRNA sequence

\begin{tabular}{|c|c|}
\hline Process & miRNA sequence \\
\hline $\begin{array}{l}\text { hsa-miR-16-5p with target } \\
\text { sequence } 5 \text { '-3, }\end{array}$ & UAGCAGCACGUAAAUAUUGGCG \\
\hline $\begin{array}{l}\text { hsa-miR-21-5p with target } \\
\text { sequence } 5 \text { '-3, }\end{array}$ & UAGCUUAUCAGACUGAUGUUGA \\
\hline $\begin{array}{l}\text { hsa-miR-29c-3p with } \\
\text { target sequence } 5 \text { '-3' }\end{array}$ & UAGCACCAUUUGAAAUCGGUUA \\
\hline $\begin{array}{l}\text { has-miR-141 with target } \\
\text { sequence } 5 \text { ' }-3 \text { ' }\end{array}$ & UAACACUGUCUGGUAAAGAUGG \\
\hline $\begin{array}{l}\text { EBV-miR-BART7-3p with } \\
\text { target sequence 5'-3' }\end{array}$ & UUCUCCGAACGUGUCACG \\
\hline
\end{tabular}

Analysis of the amplification curve was done with BioRad CFX Manager software to obtain the quantitative cycle $(\mathrm{Cq})$, quantification curve, and melting curve. The $\mathrm{Cq}$ value of each miRNA is then normalized to the reference gene to obtain the difference of $\mathrm{Cq}(\Delta \mathrm{Cq})$. The relative expression level (fold change) of NPC patients and normal subjects was calculated after the Livak's equation, Fold Change $=2^{-\Delta \Delta \mathrm{Cq}}[10]$.

\subsection{Treatment and evaluation}

The standard treatment of locally advanced NPC is chemoradiation. Patients received external radiation and concurrent chemotherapy with 5FU and cisplatin. External radiation was given 5 days a week using a fractionated dose of $200 \mathrm{cGy}$ each day for a total of $6600 \mathrm{cGy}$. Treatment response was evaluated at week-12 after treatment by performing a physical examination and computed tomography (CT) scanning.

\section{RESULT}

\subsection{Selecting a reference gene}

Preliminary results showed that miR-16, miR-21, and miR-155 were expressed in all plasma samples, while miR-145 and U6 snRNA were expressed at very low levels (graphic 1). MiR-16 was consistently showed good amplification curve and melted peaks among all samples; therefore, miR-16 was chosen as a reference for data normalization.

\subsection{Characteristics of the study subjects}

A total of 52 patients and 10 normal subjects were enrolled in this study initially. Due to a limited radiotherapy facility, only 17 patients could be treated immediately after diagnosis. Patients' mean of age was $45.1+12.53$ years, ranging from 14 to 68 years old (table 1).

\subsection{Expression levels of selected miRNAs}

MiR-21 and miR-29c were detected in all subjects (figure 2), whereas miR141 (figure 3) was detected in $22(42.3 \%)$ patients and EBV-miR-BART7 (figure 4$)$ in $26(50 \%)$ patients. The expression of all miRNA did not achieve statistical significance. The fold change of each miRNA was 0.37 for miR-21; 0.72 for miR-29c, 1.07 for miR-141, and 0.30 for miR-BART7 (table 2).

The levels of miRNA expressions were similar across the disease stage (table 3). There was no significant difference of expression of miR-21 or miR$29 \mathrm{c}$ between baseline and after standard therapy in 17 patients receiving chemoradiation (table 4). However, miR-21 tended to decrease in patients with $\mathrm{CR}$ and tend to increase in patients with PR (table 5 and graphic 5). There was a tendency that miR-29c was increased in PR patients (table 5 and graphic 6).

\section{DISCUSSION}

Plasma miRNA expressions have never been studied in Indonesia. This study is the first attempt to detect plasma miRNA in true clinical settings; in which, patients were prospectively followed from diagnosis to follow-up visits after definitive treatment.

It was successfully identified miRNA expression in plasma samples from NPC patients and normal subjects. However, while miR-21 and miR-29c were expressed in all subjects, miR-141 and miR-BART7 were expressed only in some of the patients in very small quantities $(\mathrm{Cq}>35)$.

Based on the literature study, miR-21, miR-141, and miR-BART7 are up-regulated and miR-29c is down-regulated in NPC patients compared to healthy persons. Our results showed different findings; plasma miR-21 and miR-BART7 were down-regulated, while miR-29c was only slightly down-regulated and miR-141 did not differ between NPC patients and normal subjects. However, the difference did not achieve clinical and statistical significance (fold change less than 2).

Research on miRNA expressions in NPC has just been performed in less than a decade with different results. One study using U6 as an internal control showed that miR-16 and miR-21 were the most expressed miRNA in plasma (with a fold change of 28.86 and 6.64, respectively) [11]. In addition, they found that the fold change of miR-29c and miR-141 were 0.124 and 1.140 , which were quite similar to our results.

The difference in the results could be due to the use of an internal reference gene. Previous NPC studies used U6 while this study used miR-16 as an internal control. MiR-16 was chosen because our preliminary test showed its consistent expression in all samples, whereas the expression of U6 was very low (figure 1). This study is the first to use miR-16 as a reference gene in the plasma of NPC patients.

MiR-16 and U6 are the most frequent reference genes for internal control of miRNA expression. However, both genes have some limitations. The concentration of miR-16 was vulnerable to hemolysis and could increase $\mathrm{Cq}$ [12]. Recent studies in 80 patients (20 patients in each with gastric, nasopharyngeal, colorectal, and breast cancers) showed that there was a large fluctuation of U6 expression because it was unstable during freezing and thawing process [13]

The study of miRNA expression is still in its infancy, and the results are often inconsistent. Our study showed that miR-21 expression was not increased and no other study could confirm our finding. However, under-expressed miR-21 might not be reported. In a study with 270 miRNA from NPC tissues, there was no report on miR-21 status, whether it was up- or down-regulated [14]. It might not be analyzed due to very low concentration $(\mathrm{Cq}>35)$ or fold change $<2$.

There was no difference in miRNA expression among stage II, III, and IV patients. In this study, there was no patient in stage I found, while patients with distant metastasis (stage IVC) were not enrolled. The similar miRNA expression levels among patients stage II, III, and IV indicated that at the molecular level, these patients are homogeneous. It is possible that miRNA expressions are different during the early carcinogenesis process or after distant metastasis occurs.

Study reports evaluating the relation between miRNA expression and disease stage are scarce. One of the earliest studies was the difference of miR-21 expression level between stage I and stage II-III breast cancer. In that study, the investigators found a higher expression of miR-21 in stage II-III and in patients with nodal metastasis [15]. In NPC patients, the expression of tissue miR-21 is over-expressed in stage III-IV patients, in patients with nodal metastasis and distant metastasis [16]. However, a study using plasma samples of 217 NPC patients showed different results. The expression of miR-21 tended to decrease as the tumor size increased (Spearman $\mathrm{r}=-0,159 ; \mathrm{p}=0,019)$ and nodal status increased (Spearman $\mathrm{r}=-0,160$; $\mathrm{p}=0,018)$ [11]. There was no correlation between miR-21 expression and disease stage based on the TNM system Spearman $\mathrm{r}=-0,130 ; \mathrm{p}=0,056$ ). Their results suggest that miR-21 plays a role at the early stage of the disease [11].

Many studies ofmiRNA expressions in NPC were associated with distant metastasis and survival. Among the earliest study was the expression of miR-29c, when its downregulation was associated with metastatic event [7]. MiR-29c could suppress invasion and metastasis by increasing the expression of its target, i.e., the T-cell lymphoma invasion and metastasis 1 (TIAM) 1 gene [17]. Other study found that lymphatic invasion and advanced TNM stage were correlated with the level of plasma miR-9. The expression level of miR-9 could distinguish locoregional NPC cases from metastatic NPC with high sensitivity and specificity [18]. A recent study reported that a combination of four miRNAs (miR-22, miR-572, miR-638, and miR-1234) could differentiate NPC stage and has a better prognostic role than the TNM system [19]. 
A recent study showed that the tissue level of EBV-miR-BART7 is positively related to the degree of lymph node involvement ( $N$ stage of the TNM system) and clinical stage of NPC. The mechanism by which miR-BART7 could be responsible to disease progression was shown in vitro when it specifically targets the phosphatase and tensin homolog (PTEN) gene, a major human tumor suppressor gene regulating the PI3K/AKT signaling pathway [20].

This study found no significant difference between miR-21 and miR-29c expression between before and after treatment. However, there was a tendency that the miR expression level was higher in patients with PR compared to CR. In addition, miR-21 expression tended to increase in PR and tend to decrease in CR patients, while miR-29c tended to increase in patients with PR.

Early experiments in NPC cells (CNE line) showed 44 miRNAs were expressed after a chemotherapy course with 5 -FU and cisplatin, including miR16, miR-21, and miR-29c. It was concluded that chemotherapy could alter miRNA profiles. However, almost all miRNAs, except miR-29a and 29-c, were also expressed by normal cells [21]. A recent study tested miR-21 on irradiated NPC cells (CNE-2 line). The experiment showed that miR-21 was among the most up-regulated miRNA in radioresistant cells. Radiosensitivity will increase after miR-21 was down-regulated [22].

An experimental study showed that mir-29c knockdown might cause resistance to radiotherapy and cisplatin chemotherapy through the up-regulation of antiapoptotic proteins Mcl-1 and Bcl-2. Therefore, miR-29c expression is needed to obtain a treatment response [23].

Comparing miRNA expression level before and after treatment represents how the body responds to a therapeutic intervention on an ongoing neoplastic process. However, it seems that single miRNA expression is insufficient to predict treatment response. A combination of several miRNAs might give a better prediction. For example, a study using deep sequencing technique found a combination of 4 miRNAs (miR-483-5p, miR-103, miR-29a, and let-7c) as miR-signature to predict 5-year survival of NPC patients [24]. That study select miRNA of a panel that included 146 human miRNAs, 51 new miRNAs, and 95 EBV-miRNAs. In another study, the investigators found a different miRNA set to predict NPC patients' survival, i.e., miR-22, miR-572, miR-638, and miR-1234 [19]. Both of the studies above have been validated in their institutions; therefore, it is unlikely that their results are a coincidence. None of these studies use miR-21 or miR-29c, which has been widely evaluated previously. Altogether, results from various miRNA studies in NPC might show the complexity of nasopharyngeal carcinogenesis and their alterations after therapeutic intervention.

There are some limitations to our study. First, we only included four selected miRNAs without a pilot study. These miRNAs were selected based on previous reports with convincing results on their roles in NPC patients. Second, the limited number of patients receiving standard treatment is insufficient to draw a firm conclusion on the role of miR-21 and miR-29c as predictors of treatment response. However, the results could give a foundation for further research on how to use miRNA expression in clinical settings. Third, the use of miR-16 as a reference gene could be bias because it could also be up-regulated in NPC patients [11]. Data normalization by comparing the amount of RNA included in the RT-PCR might not be right because RNA content in the plasma or serum varies according to the physical or pathological condition of the patient. Currently, there is no verified housekeeping gene in the blood that can be used for normalization [25]

\section{CONCLUSION}

From the study results, it can be concluded that miR-21 and miR-29c are expressed in all NPC patients, but miR-141 and miR-BART7 are only expressed at very low levels in some of the patients. The levels of miRNAs' expression are almost similar among stage II, III, and IV patients suggesting that at the molecular level, locally advanced NPC are homogenous. The expression of miR-21 is potentially used as a biomarker to monitor the treatment response of NPC patients.

\section{REFERENCE}

[1] Ferlay J, Shin HR, Bray F, Forman D, Mathers C, Parkin DM. Estimates of worldwide burden of cancer in 2008: GLOBOCAN 2008. International journal of cancer. 2010;127(12):2893-917.

[2] Adham M, Kurniawan AN, Muhtadi AI, Roezin A, Hermani B, Gondhowiardjo S, et al. Nasopharyngeal carcinoma in Indonesia: epidemiology, incidence, signs, and symptoms at presentation. Chinese journal of cancer. 2012;31(4):185.

[3] Wei WI, Sham JS. Nasopharyngeal carcinoma. The Lancet. 2005;365(9476):2041-54
[4] Lee YS, Dutta A. MicroRNAs in cancer. Annual Review of Pathological Mechanical Disease. 2009;4:199-227.

[5] Si M, Zhu S, Wu H, Lu Z, Wu F, Mo Y. miR-21-mediated tumor growth. Oncogene. 2007;26(19):2799.

[6] Zhu S, Wu H, Wu F, Nie D, Sheng S, Mo Y-Y. MicroRNA-21 targets tumor suppressor genes in invasion and metastasis. Cell research. 2008;18(3):350

[7] Sengupta S, den Boon JA, Chen I-H, Newton MA, Stanhope SA, Cheng Y-J, et al. MicroRNA $29 \mathrm{c}$ is down-regulated in nasopharyngeal carcinomas, up-regulating mRNAs encoding extracellular matrix proteins. Proceedings of the National Academy of Sciences. 2008;105(15):5874-8.

[8] Zhang L, Deng T, Li X, Liu H, Zhou H, Ma J, et al. microRNA-141 is involved in a nasopharyngeal carcinoma-related genes network. Carcinogenesis. 2010;31(4):559-66.

[9] Chan JY-W, Gao W, Ho W-K, Wei WI, Wong T-S. Overexpression of Epstein-Barr virus-encoded microRNA-BART7 in undifferentiated nasopharyngeal carcinoma. Anticancer research. 2012;32(8):3201-10.

[10] Livak KJ, Schmittgen TD. Analysis of relative gene expression data using real-time quantitative PCR and the 2- $\triangle \Delta C T$ method. methods. 2001;25(4):402-8.

[11] Liu X, Luo H-N, Tian W-D, Lu J, Li G, Wang L, et al. Diagnostic and prognostic value of plasma microRNA deregulation in nasopharyngeal carcinoma. Cancer biology \& therapy. 2013;14(12):1133-42.

[12] McDonald JS, Milosevic D, Reddi HV, Grebe SK, Algeciras-Schimnich A Analysis of circulating microRNA: preanalytical and analytical challenges. Clinical chemistry. 2011;57(6):833-40.

[13] Xiang M, Zeng Y, Yang R, Xu H, Chen Z, Zhong J, et al. U6 is not a suitable endogenous control for the quantification of circulating microRNAs. Biochemical and biophysical research communications. 2014;454(1):210-4.

[14] Chen H, Chen G, Chen Y, Liao W, Liu C, Chang K, et al. MicroRNA deregulation and pathway alterations in nasopharyngeal carcinoma. British journal of cancer. 2009;100(6):1002.

[15] Yan L-X, Huang X-F, Shao Q, Huang M-Y, Deng L, Wu Q-L, et al. MicroRNA miR-21 overexpression in human breast cancer is associated with advanced clinical stage, lymph node metastasis and patient poor prognosis. Rna. 2008;14(11):2348-60.

[16] Ou H, Li Y, Kang M. Activation of miR-21 by STAT3 induces proliferation and suppresses apoptosis in nasopharyngeal carcinoma by targeting PTEN gene. PloS one. 2014;9(11):e109929.

[17] Liu N, Tang L-L, Sun Y, Cui R-X, Wang H-Y, Huang B-J, et al. MiR-29c suppresses invasion and metastasis by targeting TIAM1 in nasopharyngeal carcinoma. Cancer letters. 2013;329(2):181-8.

[18] Lu J, Xu X, Liu X, Peng Y, Zhang B, Wang L, et al. Predictive value of miR-9 as a potential biomarker for nasopharyngeal carcinoma metastasis. British journal of cancer. 2014;110(2):392.

[19] Liu N, Cui RX, Sun Y, Guo R, Mao YP, Tang LL, et al. A four - miRNA signature identified from genome - wide serum miRNA profiling predicts survival in patients with nasopharyngeal carcinoma. International journal of cancer. 2014;134(6):1359-68.

[20] Cai L, Lyu X, Luo W, Cui X, Ye Y, Yuan C, et al. EBV-miR-BART7-3p promotes the EMT and metastasis of nasopharyngeal carcinoma cells by suppressing the tumor suppressor PTEN. Oncogene. 2015;34(17):2156.

[21] Zhang X, Li W. 5-Fluorouracil in combination with cisplatin alters the microRNA expression profile in the CNE nasopharyngeal carcinoma cell line. Molecular medicine reports. 2012;6(2):303-8.

[22] Zhu H, Zhu X, Cheng G, Zhou M, Lou W. Downregulation of microRNA21 enhances radiosensitivity in nasopharyngeal carcinoma. Experimental and therapeutic medicine. 2015;9(6):2185-9.

[23] Zhang J-X, Qian D, Wang F-W, Liao D-Z, Wei J-H, Tong Z-T, et al. MicroRNA-29c enhances the sensitivities of human nasopharyngeal carcinoma to cisplatin-based chemotherapy and radiotherapy. Cancer letters. 2013;329(1):91-8.

[24] Wang H-Y, Yan L-X, Shao Q, Fu S, Zhang Z-C, Ye W, et al. Profiling plasma microRNA in nasopharyngeal carcinoma with deep sequencing. Clinical chemistry. 2014;60(5):773-82.

[25] Kang K, Peng X, Luo J, Gou D. Identification of circulating miRNA biomarkers based on global quantitative real-time PCR profiling. Journal of animal science and biotechnology. 2012;3(1):4. 


\section{APPENDICES}

\section{A. TABLES}

Table 1. Characteristics of the study subjects $(n=52)$

\begin{tabular}{lcc}
\hline \multicolumn{1}{c}{ Variable } & $\begin{array}{c}\text { NPC patients } \\
(\mathbf{n = 5 2})\end{array}$ & $\begin{array}{c}\text { Normal subjects } \\
(\mathbf{n = 1 0})\end{array}$ \\
\hline Age, years (mean+SD) & $45.1+12.53$ & $38.7+14.36$ \\
Male gender (n, \%) & $34(65.4 \%)$ & $6(60.0 \%)$ \\
Stage (n, \%) & & \\
IIB & $8(15.4 \%)$ & - \\
III & $16(30.8 \%)$ & - \\
IVA & $13(25.0 \%)$ & - \\
IVB & $15(28.8 \%)$ & - \\
Histopathology (n, \%) & & - \\
Squamous cell carcinoma & $2(3.8 \%)$ & - \\
Undifferentiated carcinoma & $50(96.2 \%)$ & - \\
\hline
\end{tabular}

Table 2. Expression of plasma miRNA between NPC and normal subjects

\begin{tabular}{lrrrr}
\hline \multicolumn{1}{c}{ miRNA } & NPC $(\mathbf{n}=\mathbf{5 2})$ & Normal $(\mathbf{n}=\mathbf{1 0})$ & P Value & Fold Change \\
\hline miR-21 & $2.90 \pm 2.71$ & $1.49 \pm 0.96$ & $0.110 \mathrm{a}$ & 0.37 \\
miR-29c & $7.24 \pm 1.68$ & $6.76 \pm 2.72$ & $0.544 \mathrm{a}$ & 0.72 \\
miR 141 & $11.35(3.42-14.46)$ & $11.29 \pm 2.66$ & $0.743 \mathrm{~b}$ & 1.07 \\
miRBART7 & $11.23 \pm 3.22$ & $9.47 \pm 6.00$ & $0.340 \mathrm{a}$ & 0.30 \\
\hline $\mathrm{a}$ t-test; & & & &
\end{tabular}

${ }^{b}$ Mann-Whitney U test

Table 3. Expression levels of plasma miRNA in NPC patients based on disease stage

\begin{tabular}{lcrcc}
\hline & $\begin{array}{c}\text { Stage II } \\
(\mathbf{n = 7})\end{array}$ & $\begin{array}{c}\text { Stage III } \\
(\mathbf{n = 1 7})\end{array}$ & $\begin{array}{c}\text { Stage IV } \\
(\mathbf{n = 2 8})\end{array}$ & P* \\
\hline miR-21 & $3.52 \pm 2.00$ & $1.85 \pm 1.43$ & $3.38 \pm 3.27$ & 0.150 \\
miR-29c & $7.24 \pm 0.83$ & $7.16 \pm 1.68$ & $7.28 \pm 1.87$ & 0.977 \\
miR-141 & $12.53 \pm 1.80$ & $11.37 \pm 1.89$ & $10.59 \pm 2.61$ & 0.356 \\
miR-BART7 & $12.51 \pm 2.92$ & $11.44 \pm 3.21$ & $10.90 \pm 3.41$ & 0.734 \\
\hline${ }^{c}$ one-way ANOVA & & & &
\end{tabular}

Table 4. Comparison of miRNA expression levels in NPC patients between before and after treatment $(n=17)$

\begin{tabular}{ccccc}
\hline miRNA & $\begin{array}{c}\text { Before } \\
\text { Treatment }\end{array}$ & $\begin{array}{c}\text { After } \\
\text { Treatment }\end{array}$ & P* & Fold change \\
\hline miR-21 (mean \pm SD) & $3.86 \pm 4.08$ & $4,16 \pm 5,30$ & 0,981 & 0,98 \\
miR-29c (mean \pm SD) & $7.00 \pm 1.68$ & $7,33 \pm 2,61$ & 0,287 & 0,80 \\
\hline${ }^{c}$ Wilcoxon's signed ranks test; SD=standard deviation & &
\end{tabular}

Table 5. Comparison of miRNA expression levels between NPC patients with complete response (CR) and partial response (PR)

\begin{tabular}{lcc}
\hline & $\begin{array}{c}\text { Complete Response } \\
(\mathbf{n}=\mathbf{1 3})\end{array}$ & $\begin{array}{c}\text { Partial Response } \\
(\mathbf{n}=\mathbf{4})\end{array}$ \\
\hline miR-21 before treatment $(\operatorname{mean} \pm \mathrm{SD})$ & $4.13 \pm 3.65$ & $3.00 \pm 5.86$ \\
miR-21 after treatment $($ mean $+\mathrm{SD})$ & $2.74 \pm 3.23$ & $8.77 \pm 8.43$ \\
p value* & 0.650 & 0.465 \\
miR-29c before treatment $(\operatorname{mean} \pm \mathrm{SD})$ & $6.76 \pm 1.70$ & $7.80 \pm 8.93$ \\
miR-29c after treatment $(\operatorname{mean} \pm \mathrm{SD})$ & $6.84 \pm 2.66$ & $8.93 \pm 1.90$ \\
p value* & 0.701 & 0.068 \\
\hline${ }^{\mathrm{c}}$ Wilcoxon's signed ranks test; $\mathrm{SD}=$ standard deviat & &
\end{tabular}

\section{B. GRAPHICS}

Graphic 1. The amplification and melt curves of miR-16, miR-21, miR155 , and U6 during the preliminary study to select a reference gene

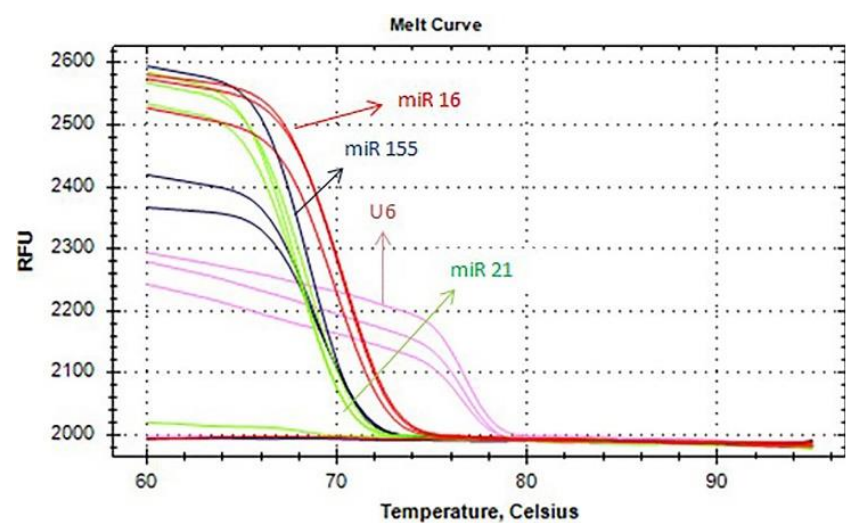

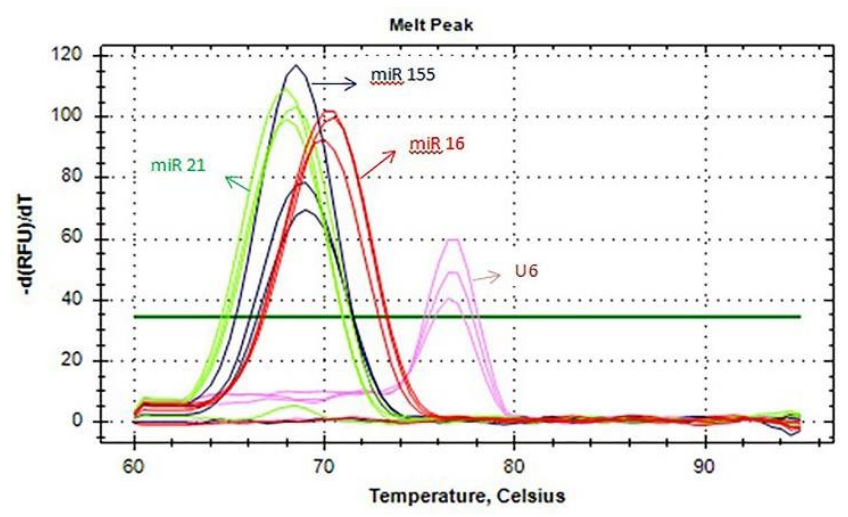

Graphic 2. The amplification and melt peak curves of miR-21 (red) and miR-c (blue) in nasopharyngeal cancer patients
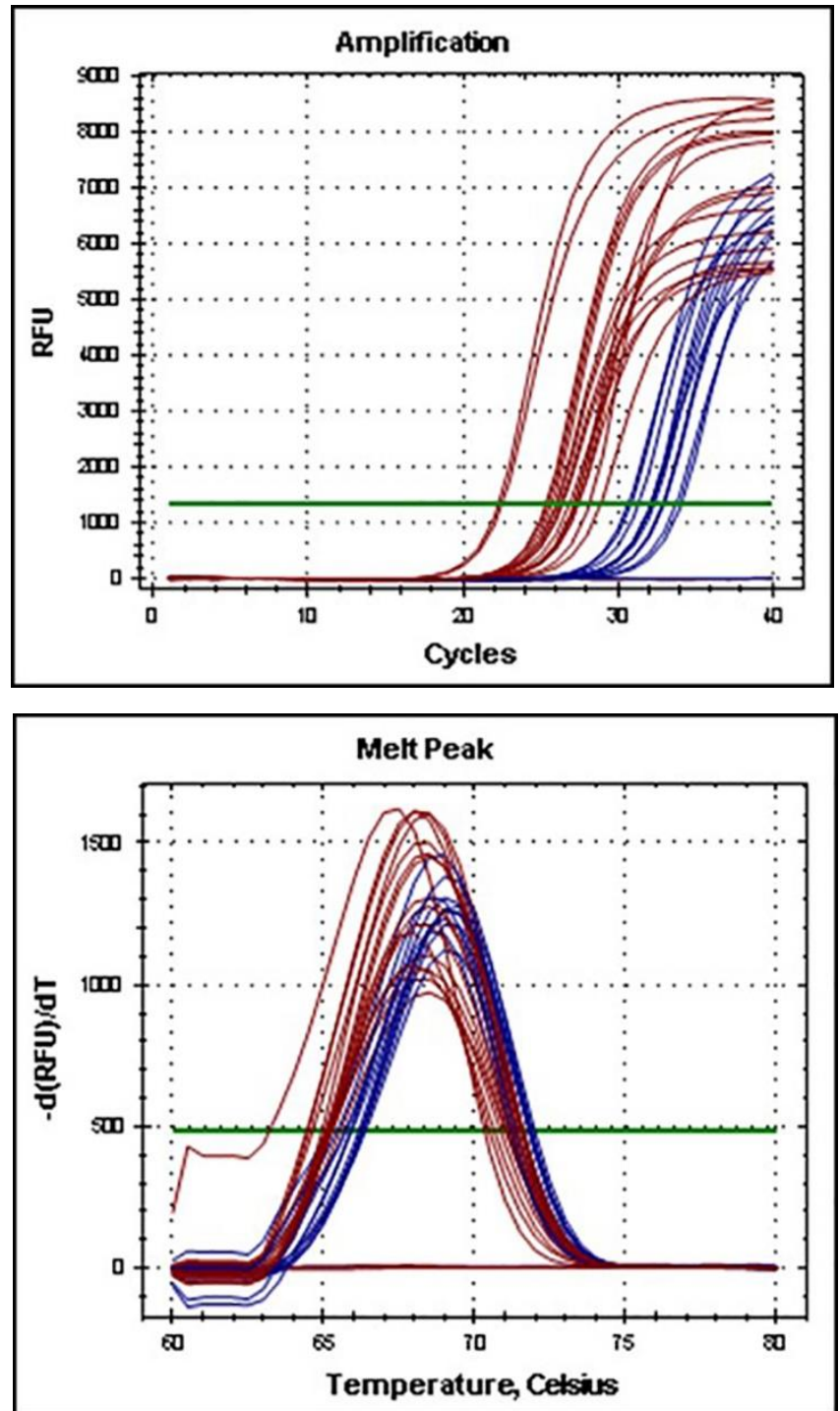
Graphic 3. The expression of miR-141 after the $30^{\text {th }}$ cycle

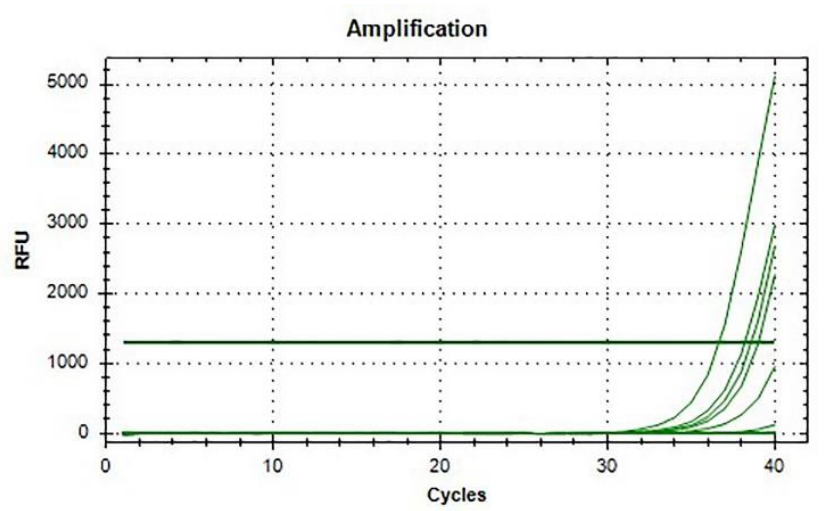

Graphic 4. The expressions of miR-BART7 in NPC patients (dark green) compared to miR-BART7 in normal subjects (blue) and to the reference gene miR16 (light green).

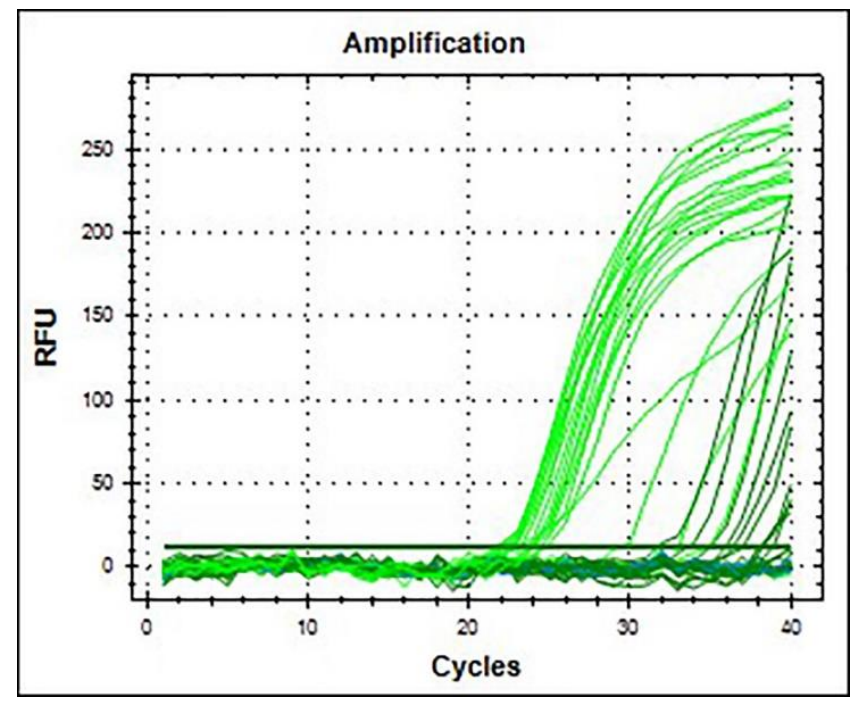

Graphic 5. The expression levels of miR-21 in patients treated with chemoradiation

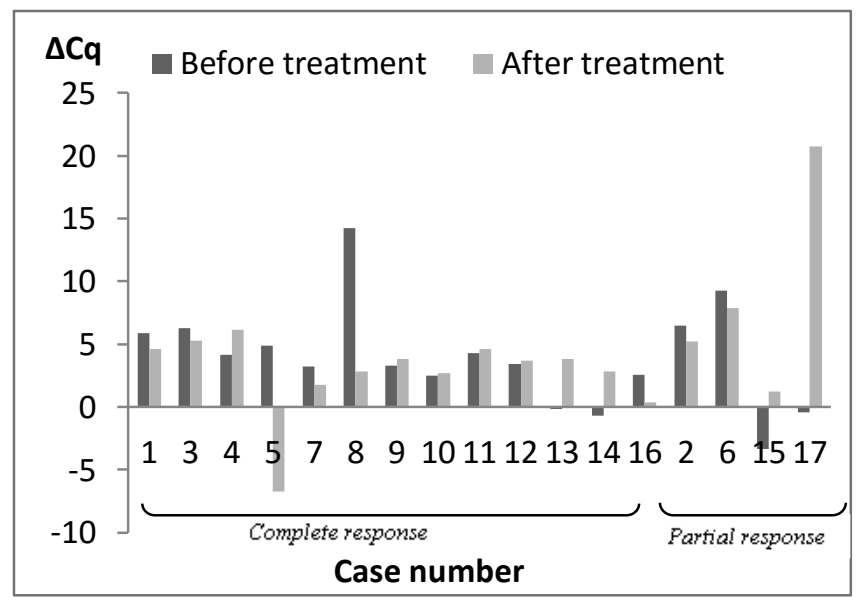

Graphic 6. The expression levels of miR-29c in patients treated with chemoradiation

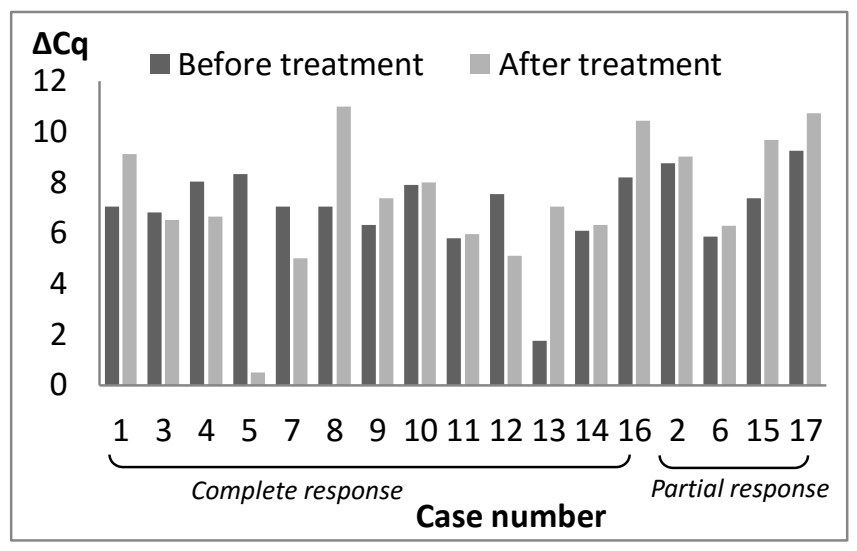

